\title{
Efectos del aislamiento social, preventivo y obligatorio en pacientes con diabetes tipo 2
}

\author{
Effects of lockdown in patients with type 2 diabetes
}

\author{
Ezequiel H. Forte ${ }^{1,2 *}$, Carolina Gómez-Martín ${ }^{3}$, Paola Harwicz², Augusto Lavalle-Cobo ${ }^{2,4}$, Diana Millán 5 , \\ Emiliano Salmeri, ${ }^{2,6}$, Carlos Buso ${ }^{2,7}$ y Hugo Sanabria ${ }^{2,8}$ \\ ${ }^{1}$ CENDIC Centro Diagnóstico Cardiovascular, Concordia, Entre Ríos; ${ }^{2}$ Consejo de Cardiometabolismo, Sociedad Argentina de Cardiología; ${ }^{3}$ Centro \\ de Endocrinología y Diabetes (CENDIA), Concordia Entre Ríos; ${ }^{4}$ Sanatorio Finochietto, Ciudad Autónoma de Buenos Aires; ${ }^{5}$ Clínica del Sol, Ciudad \\ Autónoma de Buenos Aires; ${ }^{6}$ Instituto Argentino de Diagnóstico y Tratamiento, Ciudad Autónoma de Buenos Aires; ${ }^{7}$ Consultorio Integral Diabetológico, \\ Morón; ${ }^{8}$ Instituto Cardiovascular de Buenos Aires, Ciudad Autónoma de Buenos Aires. Argentina
}

\section{Resumen}

Objetivo: Evaluar el impacto del aislamiento social, preventivo y obligatorio sobre al aumento de peso, el nivel de actividad física, la adherencia al tratamiento y la inmunización antigripal y antineumocócica en pacientes con diabetes tipo 2 (DM2). Método: Se realizó un seguimiento telefónico de pacientes con DM2 luego de al menos 100 días de comienzo del aislamiento. Se compararon dos regiones agrupadas en relación con el número de casos: región 1, más de 150 casos/100, 000 habitantes, y región 2, menos de 150 casos/100,000 habitantes. Resultados: Se contactaron 454 pacientes con DM2. El 42\% reportaron incremento de peso y el 7\% refirió haber aumentado más de $5 \mathrm{~kg}$. En relación con la actividad física, se observó un promedio más bajo en la región 1 (367.5 [0-5698] MET/sem) que en la región 2 (720 [0-7066] MET/sem) (p=0.0009). La adherencia al tratamiento farmacológico disminuyó en forma global, pero aumentó en las zonas de mayor circulación viral. Por otra parte, hubo un incremento de vacunación antigripal y antineumocócica, alcanzando coberturas del $80 \%$ y el $70 \%$, respectivamente. Conclusiones: En una población de alto riesgo cardiovascular y con una prevalencia de sobrepeso/obesidad elevada, el aislamiento social, preventivo y obligatorio se asoció con ganancia de peso y menos actividad física, lo que podría resultar deletéreo sobre la salud cardiovascular de los pacientes con DM2. Por otro lado, se observan algunos aspectos positivos, como el aumento de las inmunizaciones y el incremento de la adherencia en las zonas más afectadas.

Palabras clave: COVID-19. Diabetes mellitus. Enfermedades cardiovasculares. Pandemias.

\begin{abstract}
Objective: To evaluate the impact of lockdown on weight gain, physical activity level, adherence to treatment, and flu and pneumococcal immunization in patients with type 2 diabetes (DM2). Method: Patients with DM2 were followed by telephone after at least 100 days of the start of the lockdown. Two regions were compared, grouped in relation to the number of cases: region 1, more than 150 cases/100,000 population, and region 2, less than 150 cases/100,000 population. Results: 454 patients with DM2 were contacted. Forty-two percent of the patients reported weight gain and $7 \%$ reported having gained more than $5 \mathrm{~kg}$. In relation to physical activity, a lower average was observed in region 1 (367.5 [0-5698] MET/week) than in

\footnotetext{
Correspondencia:

*Ezequiel H. Forte

E-mail: ezeforte@yahoo.com

Fecha de recepción: 28-09-2020

Fecha de aceptación: 07-11-2020

DOI: $10.24875 /$ ACM.20000443

1405-9940 / @ 2020 Instituto Nacional de Cardiología Ignacio Chávez. Publicado por Permanyer. Este es un artículo open access bajo la licencia

Disponible en internet: 27-11-2020

Arch Cardiol Mex. 2020;90(4):1-8 www.archivoscardiologia.com CC BY-NC-ND (http://creativecommons.org/licenses/by-nc-nd/4.0/).
} 
region 2 (720 [0-7066] MET/week) ( $p=0.0009)$. Adherence to drug treatment decreased overall but increased in the areas of greatest viral circulation. On the other hand, there was an increase in influenza and pneumococcal vaccination, reaching coverage levels of $80 \%$ and $70 \%$, respectively. Conclusions: In a population at high cardiovascular risk and with a high prevalence of overweight/obesity, the lockdown was associated weight gain and lower physical activity, which could be detrimental to the cardiovascular health of patients with DM2. On the other hand, some positive aspects were observed, such as increased immunizations and increased adherence in the most affected areas.

Key words: COVID-19. Diabetes mellitus. Cardiovascular disease. Pandemics.

\section{Introducción}

Las personas con diabetes tipo 2 (DM2) tienen un elevado riesgo de presentar complicaciones derivadas de la infección por SARS-CoV-2 ${ }^{1}$. El adecuado control glucémico podría prevenir la transmisión de la COVID-192 y reducir las complicaciones en las personas con $\mathrm{DM}^{3}{ }^{3}$. En este sentido, el control integral de la DM2 requiere un alto nivel de autocuidado, un estilo de vida saludable, actividad física (AF) y adecuada adherencia al tratamiento 4 . El 20 de marzo de 2020 se declaró el aislamiento social preventivo y obligatorio (ASPO) en toda la República Argentina ${ }^{5}$. Durante los periodos de catástrofes naturales y en pandemias por enfermedades infecciosas se observan efectos deletéreos económicos y psicológicos ${ }^{6}$. Por otra parte, la falta de un seguimiento apropiado de estas patologías en tiempos de pandemia podría generar un exceso de muertes de causa cardiovascular?.

El objetivo de nuestro trabajo fue evaluar si la implementación del ASPO en el contexto de la pandemia de COVID-19 influyó en el control de salud de los pacientes con DM2, en relación con el peso, el nivel de AF, la adherencia al tratamiento y las inmunizaciones antigripal y antineumocócica.

\section{Método}

Del 2 de mayo al 30 de julio de 2019 se realizó un estudio en consultorios ambulatorios de cardiología de 17 provincias de la República Argentina. Durante los 3 meses del estudio se asistió a 1189 pacientes con diagnóstico previo de DM2. Ingresaron al registro los primeros dos pacientes de cada día de consulta (preespecificado por protocolo) que cumplieron los criterios de inclusión y aceptaron su participación, previa firma del consentimiento informado que incluía un seguimiento a 1, 3 y 5 años. La cohorte inicial de 2019 la conformaron 694 pacientes. Durante el año 2020, con más de 100 días desde el comienzo del ASPO se realizó un cuestionario por vía telefónica a los pacientes enrolados en 2019 que no presentaran síntomas ni criterios de COVID-19. EI cuestionario fue respondido por el paciente o por un familiar de primer grado. El nivel de AF se evaluó con el cuestionario validado IPAQ (International Physical Activity Questionnaire) en su versión corta ${ }^{8}$. Se definió «inactividad física» como menos de 150 minutos de AF de cualquier intensidad. Para la evaluación de la adherencia al tratamiento se usó el test de Morisky Green. El aumento de peso se definió por una pregunta de autorreporte: ¿Aumentó de peso durante la pandemia de COVID-19? La enfermedad cardiovascular (ECV) se definió como compromiso vascular carotídeo, cerebral, coronario o periférico, y complicaciones microvasculares como nefropatía, neuropatía, retinopatía y pie diabético. Se definió la inmunización como la aplicación de las vacunas antigripal y antineumocócica.

Dada la extensión de la República Argentina, la aparición desigual, progresiva y por regiones del SARS-CoV2 y las diferentes modalidades y niveles de ASPO, dividimos las regiones en Área Metropolitana de Buenos Aires/Chaco (región 1), con más de 150 casos/100.000 habitantes (163.000 personas afectadas correspondientes al $93 \%$ de los casos de todo el país), y resto del país (región 2), con menos de 150 casos/100.000 habitantes al día del cierre de la encuesta.

La región 1 quedó conformada por pacientes provenientes de Ciudad Autónoma de Buenos Aires, Tigre, Ciudad Evita, Morón, Quilmes, Boulogne, José C. Paz, Casanova, Caseros, Lomas del Mirador, Marcos Paz, Merlo, Pacheco, Padua, Palomar, Pilar, San Isidro, San Miguel, Villa Sarmiento, Villa Tesei y Chaco. La región 2 quedó conformada por pacientes provenientes de Provincia de Buenos Aires (9 de Julio, Mercedes, Bahía Blanca), Chubut, Corrientes, Entre Ríos, Formosa, Jujuy, La Pampa, Mendoza, Misiones, Neuquén, Río Negro, Santa Cruz, Tierra del Fuego y Tucumán.

En el análisis estadístico, las variables cuantitativas con distribución normal se expresaron como media y 
Tabla 1. Características basales de la población y su comparación por regiones

\begin{tabular}{|c|c|c|c|c|}
\hline Características generales & Global & Región 1 & Región 2 & $\mathbf{p}$ \\
\hline Edad en 2020 (años) & $65.4 \pm 10.5$ & $65.3 \pm 9.8$ & $65.4 \pm 10.8$ & NS \\
\hline Edad $<55$ años $(\%)$ & 14.4 & 12.3 & 15.5 & NS \\
\hline Edad 55-65 años (\%) & 24.3 & 27.8 & 22.4 & NS \\
\hline Edad 66-75 años (\%) & 30.1 & 29.6 & 30.3 & NS \\
\hline Edad > 75 años $(\%)$ & 17 & 15.4 & 17.9 & NS \\
\hline Sexo (mujeres, \%) & 41.3 & 39.5 & 43.2 & NS \\
\hline IMC en $2019\left(\mathrm{~kg} / \mathrm{m}^{2}\right)$ & $32.1 \pm 6$ & $31.7 \pm 6.4$ & $33.2 \pm 5.8$ & NS \\
\hline Evolución de DM2 en 2020 (años) & $11.8 \pm 8.6$ & $10.9 \pm 7.8$ & $12.2 \pm 9$ & NS \\
\hline Sin cobertura médica (\%) & 9.3 & 18.5 & 4.1 & 0.00 \\
\hline PAMI (\%) & 21.2 & 24.7 & 19.3 & NS \\
\hline OS o prepaga* & 69 & 56.8 & 75.8 & 0.00 \\
\hline ECV (\%) & 51.2 & 49 & 53.5 & NS \\
\hline $\operatorname{ACV}(\%)$ & 4.2 & 4.9 & 3.8 & NS \\
\hline Nefropatía (\%) & 28.4 & 29 & 28 & NS \\
\hline Retinopatía (\%) & 10 & 11.7 & 9 & NS \\
\hline Neuropatía (\%) & 9 & 8.6 & 9.3 & NS \\
\hline Pie diabético (\%) & 2.9 & 3.7 & 2.4 & NS \\
\hline Sobrepeso en 2019 (IMC 25-29, \%) & 32.2 & 29.4 & 33.6 & NS \\
\hline Obesidad en 2019 (IMC $\geq 30, \%)$ & 59.9 & 58.2 & 60.8 & NS \\
\hline
\end{tabular}

ACV: accidente cerebrovascular; DM2: diabetes mellitus tipo 2; ECV: enfermedad cardiovascular; IMC: índice de masa corporal; NS: no significativo; PAMI: Programa de Atención Médica Integral de jubilados y pensionados; OS: Obra Social Sindical.

*Prepaga: sistema de salud privado.

desviación estándar, y se analizaron mediante la prueba de Fisher. Las variables dicotómicas se expresaron como porcentajes y se analizaron mediante la prueba de ji al cuadrado. Para las datos apareados se utilizó el test de McNemar. Las variables estadísticamente significativas en el análisis univariado se analizaron mediante regresión logística para evalauar los factores asociados independientes. Se definió un valor de $p<0.05$ como estadísticamente significativo. Para el análisis se utilizó el software EPIINFO 7.

\section{Resultados}

Se llamó por teléfono a 502 pacientes ( $72 \%$ de la cohorte del 2019), de los cuales 454 respondieron el cuestionario (4 pacientes habían fallecido, 3 estaban fuera del país, 20 rechazaron responder las preguntas y 21 tenían números erróneos o incompletos). Ninguno de los pacientes contactados presentaba sintomatología ni había sufrido infección por SARS-CoV-2. El 33.7\% de los pacientes correspondían a la región 1 y el $66.3 \%$ a la región 2. El cuestionario fue respondido en el $85.3 \%$ de los casos por el paciente y en el $14.7 \%$ por un familiar de primer grado. En el momento de la evaluación, la mediana de duración del ASPO era de 113 (103-123) días. La media de edad fue de $65.4 \pm 10.5$ años y predominó el sexo masculino (66.3\%). El tiempo de evolución de la DM2 fue de $11.8 \pm 8.6$ años y el $51.2 \%$ de los pacientes tenían antecedentes de ECV. Las características generales de la muestra se exponen en la tabla 1 y las variables comparativas con el año 2019 se detallan en la tabla 2. Asimismo, en las tablas 3 a 5 se listan el incremento de peso, la AF, la adherencia al tratamiento y las inmunizaciones según grupos de edad, sexo y cobertura médica. 
Tabla 2. Comparación de los resultados de inactividad física, adherencia al tratamiento e inmunizaciones entre los años 2019 y 2020

\begin{tabular}{|l|c|c|c|}
\hline & $\mathbf{2 0 1 9}$ & $\mathbf{2 0 2 0}$ & $\mathbf{p}$ \\
\hline Inactividad física (\%) & 55.7 & 55.1 & 0.3 \\
\hline Adherencia al tratamiento (\%) & 59.4 & 52.7 & 0.003 \\
\hline Vacunación antigripal (\%) & 61 & 81 & 0.000 \\
\hline Vacunación antineumocócica (\%) & 48.8 & 68.5 & 0.000 \\
\hline
\end{tabular}

\section{Incremento de peso}

El $48.02 \%$ de los pacientes refirieron haber aumentado de peso (el $32 \% 1 \mathrm{~kg}$, el $36 \% 2-3 \mathrm{~kg}$, el $16 \%$ $3-5 \mathrm{~kg}$ y el $16 \%>5 \mathrm{~kg}$ ). El aumento promedio estimado fue de $2,4 \mathrm{~kg}$. No se hallaron diferencias significativas entre las dos regiones evaluadas (región 1 48.5\% vs. región 2 47.1\%; $p=N S$ ). Se registró una tendencia no significativa en relación con el aumento de peso y el nivel de AF: un $51.3 \%$ de los pacientes con AF baja aumentaron de peso, frente al $41.9 \%$ de los pacientes con AF alta $(p=N S)$. En el análisis multivariado para incremento de peso (edad, antecedentes cardiovascuares, región, complicaciones microvasculares, cobertura médica, AF y sexo) se correlacionó solo con la ausencia de cobertura médica (odds ratio [OR]: 2.69; intervalo de confianza del 95\% [IC95\%]: 1-3; $p=0.005)$.

\section{Actividad física}

El 55.1\% presentaba inactividad física, proporción que no varió significativamente con respecto a la observación del año 2019 (Tabla 2). La AF promedio medida por el IPAQ durante el ASPO resultó de 594 MET $\times$ min/sem, distribuida en los siguientes niveles: AF baja $50.4 \%$, AF moderada $43.2 \%$ y AF alta $6.8 \%$. En la comparación entre regiones, se observó una AF promedio significativamente más baja en la región 1, con 367,5 (0-5698) MET/sem, que en la región 2, con $720(0-7066) \mathrm{MET} / \mathrm{sem}(p=0.0009)$ (Fig. 1).

En el análisis multivariado (grupos de edad, ECV, región, complicaciones microvasculares, cobertura médica, actividad física y sexo), la AF baja se asoció de manera significativa con la región 1 (OR: 1.8; IC95\%: $1-2 ; p=0.003)$ y con el sexo femenino (OR: 1.8 ; IC95\%: $1-2 ; p=0.005)$.

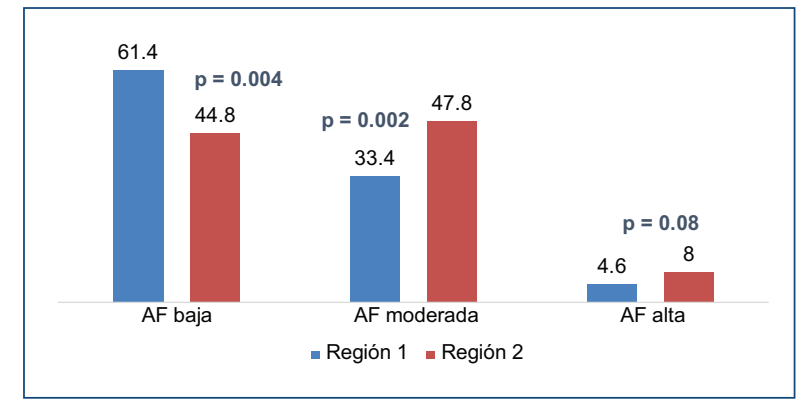

Figura 1. Nivel de actividad física (AF) de acuerdo con el IPAO según región (\%).

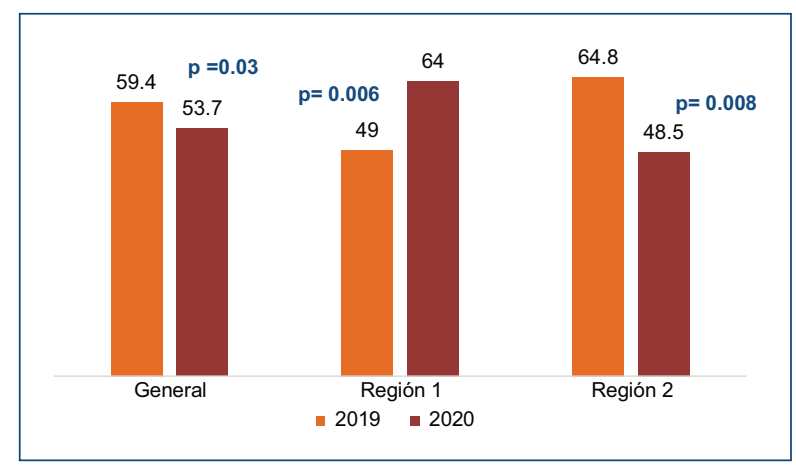

Figura 2. Adherencia al tratamiento global y discriminado por región (\%).

\section{Adherencia al tratamiento farmacológico}

Registramos una caída del $59.4 \%$ al $53.7 \%$ en el año 2020 ( $p=0.03$ ), principalmente por una disminución en la adherencia en la región 2 (Fig. 2). En el análisis multivariado (ECV, grupos de edad, región, complicaciones microvasculares, cobertura médica y sexo) observamos una asociación significativa con la región 1 (OR: 2.2; IC95\%: 1-4; $p=0.003$ ) y el sexo masculino (OR: 0.6; IC95\%: 0-4; $p=0.03$ ).

\section{Inmunizaciones}

Las coberturas de las inmunizaciones antigripal y antineumocócica aumentaron significativamente en comparación con el año 2019 (Tabla 2), sin diferencias entre las regiones evaluadas (antigripal 2020: región $183.7 \%$ vs. región $279.7 \%, p=$ NS; antineumocócica 2020: región $165.3 \%$ vs. región 2 70.4\%, p = NS). En el análisis multivariado (ECV, grupos de edad, región, complicaciones microvasculares, cobertura médica y sexo), observamos una asociación significativa en 
Tabla 3. Aumento de peso, actividad física, adherencia al tratamiento e inmunizaciones por grupos de edad

\begin{tabular}{|l|c|c|c|c|c|}
\hline & $<\mathbf{5 5}$ años & $\mathbf{5 6 - 6 5}$ años & $\mathbf{6 6 - 7 5}$ años & $>\mathbf{7 5}$ años & $\mathbf{p}$ \\
\hline Aumento de peso (\%) & 55.1 & 49.2 & 48.2 & 42.9 & 0.4 \\
\hline Actividad fisica baja (\%) & 38.5 & 52.7 & 54.7 & 54.5 & 0.03 \\
\hline Actividad física moderada (\%) & 49.2 & 39.1 & 28 & 16.8 & NS \\
\hline Actividad física alta (\%) & 12.3 & 8.2 & 5.1 & 3.9 & 0.04 \\
\hline Adherencia tratamiento (\%) & 50.8 & 50 & 59.1 & 53.2 & 0.003 \\
\hline Vacunación antigripal (\%) & 72.3 & 73.6 & 86.9 & 85.7 & 0.03 \\
\hline Vacunación antineumocócica (\%) & 55.4 & 54.5 & 80.3 & 76.6 & 0.000 \\
\hline
\end{tabular}

NS: no significativo.

Tabla 4. Aumento de peso, actividad física, adherencia al tratamiento e inmunizaciones por sexos

\begin{tabular}{|l|c|c|c|}
\hline & Hombre & Mujer & $\mathbf{p}$ \\
\hline Aumento de peso (\%) & 46 & 51 & NS \\
\hline Actividad física baja (\%) & 44.1 & 58.9 & 0.000 \\
\hline Actividad física moderada (\%) & 48.3 & 36.3 & 0.01 \\
\hline Actividad física alta (\%) & 8.4 & 4.7 & NS \\
\hline Adherencia tratamiento en $2020(\%)$ & 59.7 & 45.8 & 0.001 \\
\hline Vacunación antigripal en $2020(\%)$ & 81.7 & 80 & NS \\
\hline $\begin{array}{l}\text { Vacunación antineumocócica en } \\
2020(\%)\end{array}$ & 71.1 & 62.3 & NS \\
\hline
\end{tabular}

NS: no significativo.

relación con la región 1 (OR: 2.2; IC95\%: 1-4; $\mathrm{p}=0.003$ ) y el sexo masculino (OR: 0.6; IC95\%: 0-4; $p=0.03)$.

Cuando analizamos los subgrupos según la presencia o no de ECV no observamos diferencias significativas respecto a la ganancia ponderal $(46.6 \%$ con ECV vs. $49.5 \%$ sin ECV; $p=N S$ ) ni al alcance del objetivo de AF semanal (no alcanzaron los $150 \mathrm{~min} / \mathrm{sem}$ el $55.9 \%$ con ECV y el $54.1 \%$ sin ECV; $p=N S$ ). En los pacientes con ECV, el nivel de inactividad física fue mayor en la región 1 que en la región 2, de modo similar a lo ocurrido en la muestra global (Tabla 6).

\section{Discusión}

Nuestro estudio es el primero en Latinoamérica en evalúar el impacto del ASPO en pacientes con DM2 sobre aspectos fundamentales para el adecuado cuidado de la salud cardiovascular.

La obesidad es un factor de riesgo de complicaciones en los pacientes con COVID-19 ${ }^{9}$. Los datos sobre el incremento de peso durante el aislamiento social son escasos y variables. En Italia, en la población en general, se observó un incremento de peso del $47.5 \%{ }^{10}$. Dos estudios en pacientes con diabetes mostraron, en India, un incremeto de peso del $19 \%{ }^{11}$, y en Turquía no se registraron cambios significativos ${ }^{12}$. Casi la mitad de la población evaluada en nuestro estudio reportó haber incrementado su peso, lo cual podría generar un peor control metabólico y un exceso de muertes prevenibles ${ }^{13}$. El promedio de incremento de peso observado en nuestro estudio fue mayor que el de un reporte italiano en pacientes seguidos en una unidad de obesidad ${ }^{14}$. Las diferencias encontradas podrían deberse a la población estudiada, al número de pacientes y a diferencias socioculturales. Durante periodos de confinamiento aumenta el consumo de alimentos poco saludables asociado a la ansiedad del aislamiento $0^{15}$, el incremento de la sensación de apetito ${ }^{16}$ y el consumo de bebidas alcohólicas ${ }^{17}$. En nuestra muestra, casi la mitad de los pacientes presentaban ECV; en este sentido, la fluctuación del peso podría considerarse un factor independiente de incremento de la mortalidad en estos pacientes ${ }^{18}$. El hallazgo de la falta de cobertura médica relacionada con el aumento de peso se encuentra en línea con el bajo nivel socioeconómico observado como determinante de malnutrición en Latinoamérica ${ }^{19}$.

La AF regular disminuye la mortalidad cardiovascular y mejora el sistema inmunitario frente a posibles infecciones respiratorias ${ }^{20}$. Dos estudios en población general evaluaron el nivel de AF durante el confinamiento: 
Tabla 5. Aumento de peso, actividad física, adherencia al tratamiento e inmunizaciones según cobertura médica

\begin{tabular}{|c|c|c|c|c|}
\hline & Sin cobertura & PAMI & OS/prepaga* & $\mathbf{p}$ \\
\hline Aumento de peso (\%) & 69 & 49 & 45 & 0.002 \\
\hline Actividad física baja (\%) & 50 & 49 & 50.1 & NS \\
\hline Actividad física moderada (\%) & 45.2 & 45.8 & 42.3 & NS \\
\hline Actividad física alta (\%) & 4.7 & 5.2 & 5.7 & NS \\
\hline Adherencia tratamiento en $2020(\%)$ & $33.3 \%(14)$ & $47.9 \%(46)$ & $58.5 \%(183)$ & 0.001 \\
\hline Vacunación antigripal en 2020 (\%) & 87.5 & 83.3 & 78.9 & 0.03 \\
\hline Vacunación antineumocócica en 2020 (\%) & 47.6 & 71.9 & 70.6 & 0.001 \\
\hline
\end{tabular}

NS: no significativo; OS: Obra Social Sindical; PAMI: Programa de Atención Médica Integral de jubilados y pensionados.

*Prepaga: sistema de salud privado.

Tabla 6. Análisis de pacientes $(n=236)$ con enfermedad cardiovascular en relación con el aumento de peso, la inactividad física y el IPAO

\begin{tabular}{|l|c|c|c|}
\hline & Región 1 & Región 2 & p \\
\hline Aumento de peso & $44 \%$ & $47.8 \%$ & NS \\
\hline Inactividad física & $70.7 \%$ & $49.1 \%$ & 0.0009 \\
\hline $\begin{array}{l}\text { Suma IPAO (mediana, MET/ } \\
\text { semana) }\end{array}$ & 367.5 & 712.3 & 0.006 \\
\hline
\end{tabular}

NS: no significativo.

uno de ellos, en población italiana, mostró un incremento del nivel de AF baja de $23 \%$ al $39,6 \%{ }^{21}$; en el segundo estudio, una encuesta multicéntrica, se observó una reducción de la $\mathrm{AF}$ global del $38 \%^{22}$. En nuestro estudio, más de la mitad de los pacientes eran físicamente inactivos en 2019, porcentaje que se mantuvo durante el tiempo de ASPO. Sin embargo, al analizar el nivel de AF por regiones, en la región 1 la AF baja resultó significativamente superior que en la región 2 , lo que refleja el posible impacto de un aislamiento social mas estricto. Resultados similares se obtuvieron en un estudio multicéntrico con datos de una aplicación para teléfonos celulares, que mostró una caída más marcada del conteo de pasos según el grado de aislamiento implementado: del $48.7 \%$ en Italia, donde se declaró un bloqueo nacional, y del $6.9 \%$ en Suecia, donde se abogó por el distanciamiento social sin aislamiento estricto ${ }^{23}$. Por último, el sexo femenino se asoció de manera significativa con AF baja en el análisis multivariado, en concordancia con muchos estudios que muestran disparidad por sexo en el nivel de $\mathrm{AF}^{24}$.
La adherencia al tratamiento se encuentra en relación con diferentes factores: socioeconómicos, del tratamiento, de la enfermedad y del sistema de salud. En un estudio realizado en la Ciudad de México durante la pandemia de COVID-19 se observó una caída del $19 \%$ en la adherencia y un incremento del $22 \%$ en los problemas relacionados con el acceso al tratamiento farmacológico ${ }^{25}$. En la misma línea, en nuestro estudio hallamos una reducción significativa de la adherencia al tratamiento en comparación con el año 2019. Sin embargo, cuando comparamos por regiones, en las zonas con mayor cantidad de casos de COVID-19 (región 1) se registró una mayor adherencia. Un estudio en población alemana registró un incremento en la venta de fármacos cardiovasculares, lo que sugiere que el miedo generado por la pandemia podría tener un efecto positivo en la adherencia al tratamiento ${ }^{26}$, hecho que podría explicar los hallazgos en nuestro estudio.

Algunos datos provenientes de la experiencia en Italia podrían vincular la vacunación antigripal con un efecto protector frente a la enfermedad por SARSCoV-2 ${ }^{27,28}$. En nuestro estudio, la cobertura de vacunación se incrementó durante el ASPO, ampliando significativamente la inmunización en ambas zonas. En Argentina, el inicio de la pandemia coexistió con el periodo invernal. En el contexto actual, la sensibilidad y el miedo a padecer un cuadro infeccioso han podido incrementar el impacto de las campañas realizadas por los distintos organismos de salud y aumentar la adherencia a las indicaciones de inmunización. La mayor aplicación de la vacuna contra la gripe podría facilitar el manejo de los brotes respiratorios en escenarios 
donde los recursos y la capacidad de detección podrían ser reducidos ${ }^{29}$.

Como limitaciones del presente estudio podemos mencionar que los datos fueron autorreportados. Por otra parte, se incluyeron pacientes que consultaron a un especialista en cardiología y que podrían no representar el universo de pacientes con DM2. Finalmente, el criterio de inclusión de por lo menos 100 días de ASPO fue cumplido por todos los pacientes, pero se detecta una variabilidad en el tiempo de exposición a este. Como el ASPO aún está en curso (octubre de 2020), no podemos en este momento reportar la evaluación final de las consecuencias de un ASPO más prolongado.

\section{Conclusiones}

En una población de alto riesgo cardiovascular y con una prevalencia de sobrepeso/obesidad elevada, el ASPO se asoció a ganacia de peso y menor nivel de $A F$, lo que podría resultar deletéreo sobre la salud cardiovascular de los pacientes con DM2. Por otro lado, se observan algunos aspectos positivos, como el aumento de las inmunizaciones y el incremento de la adherencia al tratamiento en las zonas más afectadas. Consideramos importante implementar estrategias que permitan mitigar el impacto sobre los aspectos negativos de la pandemia y del ASPO, en especial en las poblaciones de mayor riesgo cardiovascular.

\section{Grupo investigadores cardiometabolismo}

Dr. Hugo Sanabria, Dra. Paula Peres Terns, Dra. Constanza Lagos, Dr. Federico Graziano, Dr. Ricardo Sebastian Galdeano, Dr. Jorge Veron, Dr. Carlos Pedroza, Dr. Eduardo Blumberg, Dr. Augusto Lavalle Cobo, Dr. Fernando Gonzalez Pardo, Dr. Carlos Klein, Dr. Juan Carlos Sendoya, Dra. Diana Millan, Dr. Juan Navarro, Dr. Cesar Damián Berestein, Dra. Paola Harwicz, Dr. Ezequiel Schnyder, Dra. Daniela Luconi, Dra. Maria del Mar Baez Nuñez, Dr. Salmeri Emiliano, Dra. Graciela Bruna, Dr Damián Ezequiel Holownia, Dr. Leonel Degenhardt, Dr. Julio Napoli, Dr. Pedro Becerra, Dr. Fabio Ruso, Dr. Juan Pablo Bonifacio, Dr. Schiavone Leonardo, Dr. Facundo laconis, Dra. Viginia Barba, Dra Florencia Sobrino, Dr. Sebastian Raul Fonseca, Dra. Duczynski Paula, Dr. Oscar Voguelman, Dr. Forte Ezequiel, Dra. Guillermina Eleit, Dra. Ana Maria Grassani, Dr. Cesar Geovanny, Dr. Carlos Javier Buso, Dr. Courtade Iriarte Pablo, Dr. Horacio Buffa, Dr. Pedro Zanardo, Dr. Natalia Prum, Dr. Raul
Maltez, Dr. Santiago Lynch, Dra. Marina Gonzalez, Dra. Valeria Varone, Dr. Alfredo Alejandro Romano.

\section{Conflictos de intereses}

Ninguno.

\section{Financiación}

La presente investigación no ha recibido ninguna beca específica de agencias de los sectores público, comercial o sin ánimo de lucro.

\section{Responsabilidades éticas}

Protección de personas y animales. Los autores declaran que para esta investigación no se han realizado experimentos en seres humanos ni en animales.

Confidencialidad de los datos. Los autores declaran que en este artículo no aparecen datos de pacientes.

Derecho a la privacidad y consentimiento informado. Los autores han obtenido el consentimiento informado de los pacientes y/o sujetos referidos en el artículo. Este documento obra en poder del autor de correspondencia.

\section{Bibliografía}

1. Apicella M, Campopiano MC, Mantuano M, Mazoni L, Coppelli A, Del Prato S. COVID-19 in people with diabetes: understanding the reasons for worse outcomes. Lancet Diabetes Endocrinol. 2020;8:782-92.

2. Wang A, Zhao W, Xu Z, Gu J. Timely blood glucose management for the outbreak of 2019 novel coronavirus disease (COVID-19) is urgently needed. Diabetes Res Clin Pract. 2020;162:108118.

3. Zhu L, She ZG, Cheng X, Qin JJ, Zhang XJ, Cai J, et al. Association of blood glucose control and outcomes in patients with COVID-19 and pre-existing type 2 diabetes. Cell Metab. 2020;31:1068-77.e3.

4. American Diabetes Association. 5. Lifestyle Management: Standards of Medical Care in Diabetes - 2019. Diabetes Care. 2019;42:S46-60.

5. Naciones Unidas - Argentina. Análisis inicial de las Naciones Unidas COVID-19 en Argentina: impacto socioeconómico y ambiental. Actualizado al 19/06/2020. Disponible en: https://www.onu.org.ar/stuff/Informe-COVID-19-Argentina.pdf.

6. Kehlenbrink S, Smith J, Ansbro E, Fuhr DC, Cheung A, Ratnayake R, et al. The burden of diabetes and use of diabetes care in humanitarian crises in low-income and middle-income countries. Lancet Diabetes Endocrinol. 2019;7:638-47.

7. Lamelas P, Botto F, Pedernera G, Alves De Lima A, Costabel JP, Belardi J. Enfermedad cardiovascular en tiempos de COVID-19. Medicina (B Aires). 2020;80:248-52.

8. Booth ML. Assessment of physical activity: an international perspective. Res Q Exerc Sport. 2000;71:s114-20.

9. Yang J, Hu J, Zhu C. Obesity aggravates COVID-19: a systematic review and meta-analysis. J Med Virol. 2020 Jun 30:10.1002/jmv.26237. doi: 10.1002/jmv.26237. Online ahead of print.

10. Di Renzo L, Gualtieri P, Pivari F, Soldati L, Attinà A, Cinelli G, et al. Eating habits and lifestyle changes during COVID-19 lockdown: an Italian survey. J Transl Med. 2020;18:229.

11. Ghosh A, Arora B, Gupta R, Anoop S, Misra A. Effects of nationwide lockdown during COVID-19 epidemic on lifestyle and other medical issues of patients with type 2 diabetes in north India. Diabetes Metab Syndr. 2020;14:917-20.

12. Önmez A, Gamsızkan Z, Özdemir Ş, Kesikbaş E, Gökosmanoğlu F, Torun S, et al. The effect of COVID-19 lockdown on glycemic control in 
Arch Cardiol Mex. 2020;90(4)

patients with type 2 diabetes mellitus in Turkey. Diabetes Metab Syndr. 2020;14:1963-6.

13. Chandrasekaran B, Ganesan TB. Sedentarism and chronic disease risk in COVID 19 lockdown - a scoping review. Scott Med J. $2020 \mathrm{Ju}$ 27;36933020946336. doi: 10.1177/0036933020946336. Online ahead of print.

14. Pellegrini M, Ponzo V, Rosato R, Scumaci E, Goitre I, Benso A, et al. Changes in weight and nutritional habits in adults with obesity during the "lockdown" period caused by the COVID-19 virus emergency. Nutrients. 2020;12:2016.

15. Ruiz-Roso MB, Knott-Torcal C, Matilla-Escalante DC, Garcimartín A Sampedro-Nuñez MA, Dávalos A, et al. COVID-19 lockdown and changes of the dietary pattern and physical activity habits in a cohort of patients with type 2 diabetes mellitus. Nutrients. 2020;12:2327.

16. Di Renzo L, Gualtieri $P$, Pivari $F$, Soldati L, Attinà A, Cinelli $G$, et al Eating habits and lifestyle changes during COVID-19 lockdown: an Italian survey. J Transl Med. 2020;18:1-15.

17. Pellechia T. Nielsen says beverage alcohol retail sales are soaring during the crisis. Forbes; 2020. Disponible en: https://www.forbes.com/sites/ thomaspellechia/2020/03/25/nielsen-says-beverage-alcohol-retail-sales-are-soaring-during-th e-crises/\#15d94451244.

18. Bangalore S, Fayyad R, Laskey R, DeMicco DA, Messerli FH, Waters DD. Body-weight fluctuations and outcomes in coronary disease. N Engl $J$ Med. 2017;376:1332-40

19. Mujica OJ, Victora CG. Obesity inequality among adults in Latin America and the Caribbean. Lancet Glob Health. 2019;7:e1589-90.

20. Nieman DC. Coronavirus disease-2019: a tocsin to our aging, unfit, corpulent, and immunodeficient society. J Sport Health Sci. 2020;9: 293-301.

21. Maugeri G, Castrogiovanni P, Battaglia G, Pippi R, D’Agata V, Palma A et al. The impact of physical activity on psychological health during $\mathrm{Co}$ vid-19 pandemic in Italy. Heliyon. 2020;6:e04315.
22. Ammar A, Brach M, Trabelsi K, Chtourou H, Boukhris O, Masmoudi L, et al. Effects of COVID-19 home confinement on eating behaviour and physical activity: results of the ECLB-COVID19 International Online Survey. Nutrients. 2020;12:1583.

23. Tison GH, Avram R, Kuhar P, Abreau S, Marcus GM, Pletcher MJ, et al. Worldwide effect of COVID-19 on physical activity: a descriptive study. Ann Intern Med. 2020;173:767-70.

24. Lounassalo I, Salin K, Kankaanpää A, Hirvensalo M, Palomäki S, Tolvanen $A$, et al. Distinct trajectories of physical activity and related factors during the life course in the general population: a systematic review. BMC Public Health. 2019;19:271.

25. Silva-Tinoco R, González-Cantú A, de la Torre-Saldaña V, Guzmán-Olvera E, Cuatecontzi-Xochitiotzi T, Castillo-Martínez L, et al. Effect in self-care behavior and difficulties in coping with diabetes during the COVID-19 pandemic. Rev Mex Endocrinol Metab Nutr. 2020;7. Disponible en: https://www. revistadeendocrinologia.com/files/rme_063_20_effect_silva_ao_uk-3.pdf

26. Kostev K, Lauterbach S. Panic buying or good adherence? Increased pharmacy purchases of drugs from wholesalers in the last week prior to Covid-19 lockdown. J Psychiatr Res. 2020;130:19-21.

27. Marín-Hernández D, Schwartz RE, Nixon DF. Epidemiological evidence for association between higher influenza vaccine uptake in the elderly and lower COVID-19 deaths in Italy. J Med Virol. 2020 Jun 4;10.1002/ jmv.26120. doi: 10.1002/jmv.26120. Online ahead of print.

28. Fink G, Orlova-Fink N, Schindler T, Grisi S, Ferrer AP, Daubenberger C, et al. Inactivated trivalent influenza vaccine is associated with lower mortality among Covid-19 patients in Brazil. medRxiv; 2020. Disponible en: https://www.medrxiv.org/content/10.1101/2020.06.29.2014 $2505 \mathrm{v} 1$.

29. Li Q, Tang B, Bragazzi NL, Xiao Y, Wu J. Modeling the impact of mass influenza vaccination and public health interventions on COVID-19 epidemics with limited detection capability. Math Biosci. 2020;325:108378. 